\title{
Should we trust abstracts from pain conferences? Publication bias and discordance between abstract and publication
}

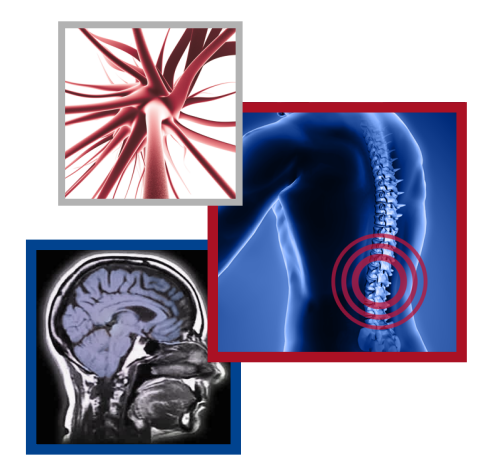

\author{
Livia Puljak*,1 \& Lenko Saric ${ }^{2}$ (D) \\ ${ }^{1}$ Center for Evidence-Based Medicine \& Health Care, Catholic University of Croatia, Zagreb, Croatia \\ ${ }^{2}$ Department of Anesthesiology \& Intensive Care Medicine, University Hospital Split, Split, Croatia \\ *Author for correspondence: Tel.: +385 (0) 137066 00; Fax: +385 (0) 137066 01; livia.puljak@gmail.com
"conference abstracts presenting the highest levels of evidence at the largest global pain congress are not necessarily dependable"

First draft submitted: 8 October 2019; Accepted for publication: 18 November 2019; Published online: 19 December 2019

Keywords: conference abstracts $\bullet$ methodology $\bullet$ pain $\bullet$ publication bias $\bullet$ reporting

Health professionals and researchers attend conferences to obtain new knowledge and to learn about results of new studies that have not yet been published in scholarly publications. However, it has been shown in various fields that conference abstracts are not necessarily dependable sources of information. One way of assessing dependability of information presented at conferences is to compare information presented in a conference abstract with information reported in a subsequent full-text publication. Authors from various clinical areas have conducted such comparisons and showed that there was a high prevalence of discrepancies between conference abstracts and journal publication [1-6].

Our team has conducted such analysis for abstracts presented at World Congress of Pain (WCP), the largest global conference in the field of pain. Our analyses were focused on studies that are considered the highest levels of hierarchy of evidence in medicine - randomized controlled trials (RCTs) [7] and systematic reviews (SRs) [8].

In the first analysis, we assessed RCT abstracts presented at four consecutive WCPs that were organized biannually from 2008 to 2014; we analyzed whether they were published in a scholarly journal, quality of their reporting and whether there was any discordance between conference abstracts and journal publications. Of the 614 analyzed RCT abstracts, about half were published by August 2016; there was no difference in publication rate of the oldest analyzed conference compared with the most recent included conference. We analyzed in detail 298 pairs of abstracts and corresponding publications and found at least some kind of discordance in a third of those pairs. We categorized discordance as qualitative and quantitative. Quantitative discordance was defined as a change in magnitude but not in direction of effect while qualitative discordance was defined as a change in direction of effect.

An example of a qualitative discrepancy would be the following: an abstract reported that the effect was slightly larger in the experimental group, but that there was no statistical difference and the manuscript claims that the effect was significantly larger in the experimental group. In such cases, implications for the pain field are major, because the abstract gives completely different conclusion for that specific outcome compared with the manuscript.

An example of a quantitative discrepancy would be, for example, providing different numerical results in the abstract, compared with the manuscript. In this case, implications for the pain field depend on the magnitude of the effect reported in manuscript and abstract; a minor difference would not have major implications, but major difference in these numbers, despite the fact that there is no difference between abstract and manuscript in direction of effect, can give a completely different message to the readers, depending on the information source they will use - an abstract or a manuscript. 
The majority of discordances we found were quantitative. A particular problem was found in the pairs where abstracts indicated that they were presenting preliminary or interim data. In those abstracts and publications, we found some kind of discordance in $79 \%$ of pairs; in five of those pairs (28\% of all preliminary abstracts), we found qualitative discordance. This means that if a reader is reading an abstract presenting preliminary/interim results, every third abstract will contain erroneous information.

Among pairs where abstracts did not indicate that they presented preliminary/interim results, we found discrepancies in a third of the pairs and qualitative discordance was found in 18 pairs $(6 \%)$, indicating that nonpreliminary abstracts contain erroneous information in one out of 16 cases. These results indicate that extreme caution needs to be taken when making decisions about health and clinical judgments based on data presented at conferences, particularly when authors are presenting results that are labelled as preliminary or interim [7].

Since multiple studies in various clinical fields studied the same issue, whether RCTs presented as abstracts at conferences were subsequently published and whether they contained dependable information, as a follow-up study we wanted to explore the same issues among abstracts of SRs presented at WCPs. Publication bias and dependability of SR conference abstracts has received very little attention in the literature so far.

Compared to RCT abstracts, fewer SR abstracts were presented at WCPs; 143 such abstracts were presented in five WCPs that took place from 2008 to 2016. In this follow-up study, we included one additional conference, compared with the previous study.

Among the 143 analyzed SR conference abstracts, we found full-text publication for 63\%. We found at least some type of discordance in $40 \%$ of abstract-publication pairs, with qualitative discordance in $16 \%$ of pairs. This indicates that one in six SR abstracts had major differences in the direction of results by the time the study reached publication [7].

In both studies, we found that reporting quality of analyzed abstracts was not optimal. We assessed reporting quality of RCT abstracts by comparing them with the reporting checklist CONSORT for Abstracts, which was published in 2008 and has 17 items [9]. We found that the analyzed RCT abstracts were inadequately reported, as the mean adherence for all domains was 26\%. We found the highest adherence (adequate in more than $90 \%$ of RCT abstracts) for checklist domains describing interventions, objectives and the conclusions.

We evaluated SR abstracts against PRISMA for Abstracts, which was published in 2013 and has 12 items [10] and the median adherence to the checklist, for all items, was $33 \%$. Since some of the conferences that we analyzed took place before 2013, we also conducted a subgroup analyses for conferences organized before the checklist publication and those organized afterward. For nine items, there were no differences in SR conference abstracts' reporting before and after checklist publication. We found differences in three items - reporting of strengths and limitations of the study significantly improved after the checklist publication, while items describing eligibility criteria and funding were reported significantly better before release of the checklist [8].

While conference organizers cannot influence dependability of information presented in the abstracts, a feasible and easily implemented intervention would be to mention reporting checklists for abstracts in instructions for authors of those abstracts. In this way, conference attendees would become aware of the existence of these checklists, in case they were not aware of them before.

\section{Conclusion}

In conclusion, our studies have shown that conference abstracts presenting the highest levels of evidence at the largest global pain congress are not necessarily dependable (i.e., authors often change their results subsequently in a journal publication). These results are in line with other studies that have analyzed dependability and publication bias of conference abstracts. Users who rely on conference abstracts for obtaining new information should be aware of this problem. Furthermore, conference abstracts are often not adequately reported and this is something that should change in the future, to enable readers to get sufficient information for such abbreviated research reports. 


\section{Financial \& competing interests disclosure}

The authors have no relevant affiliations or financial involvement with any organization or entity with a financial interest in or financial conflict with the subject matter or materials discussed in the manuscript. This includes employment, consultancies, honoraria, stock ownership or options, expert testimony, grants or patents received or pending, or royalties.

No writing assistance was utilized in the production of this manuscript.

\section{References}

1. Wieser M, Braun C, Moens Y. Publication rate of studies presented at veterinary anaesthesia specialty meetings during the years 2003-2008. Vet. Anaesth. Analg. 43(2), 118-127 (2015).

2. Hopewell S, Boutron I, Altman DG, Ravaud P. Deficiencies in the publication and reporting of the results of systematic reviews presented at scientific medical conferences. J. Clin. Epidemiol. 68(12), 1488-1495 (2015).

3. Livas C, Pandis N, Ren Y. Full-text publication of abstracts presented at European Orthodontic Society congresses. Eur. J. Orthod. 36(5), 569-575 (2014).

4. Chan KK, Siu E, Mozessohn L, Cheung MC. Publication patterns of cancer cost-effectiveness studies presented at major conferences. Curr. Oncol. 20(6), 319-325 (2013).

5. Weintraub WH. Are published manuscripts representative of the surgical meeting abstracts? An objective appraisal. J. Pediatr. Surg. 22(1), 11-13 (1987).

6. Saldanha IJ, Scherer RW, Rodriguez-Barraquer I, Jampel HD, Dickersin K. Dependability of results in conference abstracts of randomized controlled trials in ophthalmology and author financial conflicts of interest as a factor associated with full publication. Trials 17(1), 213 (2016).

7. Saric L, Vucic K, Dragicevic K et al. Comparison of conference abstracts and full-text publications of randomized controlled trials presented at four consecutive World Congresses of Pain: reporting quality and agreement of results. Eur. J. Pain 23(1), 107-116 (2019).

8. Saric L, Dosenovic S, Saldanha IJ, Kadic AJ, Puljak L. Conference abstracts describing systematic reviews on pain were selectively published, not reliable, and poorly reported. J. Clin. Epidemiol. 117, 1-8 (2019).

9. Hopewell S, Clarke M, Moher D et al. CONSORT for reporting randomised trials in journal and conference abstracts. Lancet 371(9609), 281-283 (2008).

10. Beller EM, Glasziou PP, Altman DG et al. PRISMA for abstracts: reporting systematic reviews in journal and conference abstracts. PLoS Med. 10(4), e1001419 (2013). 